\title{
Propuesta de estrategia académica basada en la deontología profesional para fortalecer las competencias axiológicas
}

\author{
Proposal academic strategy based on professional deontology to strengthen axiological \\ competences
}

\begin{tabular}{|rrr|}
\hline Yerardo Ortiz & Deivid Vargas & Luisedgar Velásquez \\
\hline yerardoortiz@gmail.com & deivi_armando@hotmail.com & luisedgarjvo@gmail.com \\
ORCID: 0000-0001-8189-1500 & ORCID: 0000-0001-7503-7922 & ORCID: 0000-0002-8368-4019 \\
& & Universidad de Carabobo, Venezuela \\
\hline
\end{tabular}

Artículo recibido en enero 2019 / Arbitrado en febrero 2019 / Publicado en mayo 2019

RESUMEN

Palabras clave:

ABSTRACT

Keywords:
La presente investigación surgió en vista de la necesidad de incorporar en el pensum de estudios de la carrera de Contaduría Pública de la Facultad de Ciencias Económicas y Sociales de la Universidad de Carabobo, una materia sobre Deontología Profesional, con el fin de formar a los estudiantes de manera integral con sólidos conocimientos y valores para afrontar el campo de trabajo con profesionalidad. Se planteó como objetivo primordial diseñar una estrategia académica para fortalecer el dominio de conocimiento en Deontología Profesional a los estudiantes de Contaduría Pública de la Universidad de Carabobo. La investigación asumió el enfoque cuantitativo, enmarcado en un diseño de campo y carácter descriptivo, apoyada con referencias bibliográficas-documentales. Como población objetivo, se enfocó en los estudiantes del décimo semestre de Contaduría Pública de la FACES-UC. A partir de ello se pudo concluir que la mayoría de los estudiantes de la carrera de Contaduría Pública de la FACES - Universidad de Carabobo no manejan ni conocen que es la deontología profesional, así como los códigos deontológicos y su importancia dentro del ejercicio profesional de la Contaduría Pública, lo cual lo limita en la realización de un ejercicio responsable, justo y honesto.

Estrateqia Académica, Deontoloaía, Ejercicio profesional, Contaduría Pública

This research arose in view of the need to incorporate in the curriculum of the Public Accounting career of the Faculty of Economic and Social Sciences of the University of Carabobo, a subject on Professional Deontology, in order to train students comprehensively with solid knowledge and values to face the field of work with professionalism. The main objective was to design an academic strategy to strengthen the domain of knowledge in Professional Deontology for students of Public Accounting at the University of Carabobo. The research assumed the quantitative approach, framed in a field design and descriptive character, supported by bibliographic-documentary references. As a target population, it focused on students in the tenth semester of Public Accounting at FACES-UC. From this, it was possible to conclude that most of the students of the Public Accounting career at FACES - University of Carabobo do not handle or know what professional deontology is, as well as the deontological codes and their importance within the professional practice of the Public Accounting, which limits it in carrying out a responsible, fair and honest exercise.

Academic Strategy, Deontology, Professional Practice, Public Accountin 


\section{INTRODUCCIÓN}

La Contaduría Pública es una profesión libre que puede ser ejercida de manera dependiente e independiente; adquiriendo así una responsabilidad aun mayor porque es una de las actividades que requiere de un mayor grado de objetividad y confianza ya que es de gran importancia tanto económica como financieramente. En la mayoría de las entidades, se necesita un Contador Público que controle, supervise y asesore en todas las actividades financieras de la organización, con el propósito de alcanzar los objetivos trazados, por lo que se hace imperante que el Contador sea un profesional con altos valores éticos y morales; ya que, su actuación y desempeño es de gran importancia para la toma de decisiones de la organización.

Es por ello, que el profesional en la carrera Contaduría Pública, debe cumplir con una serie de requerimientos para ejercer de la mejor manera sin dañar ni perjudicar a terceros con él trabajo que el mismo realiza, así pues basándonos en la teoría sobre la deontología, los requerimientos que hacen vida en esta profesión están enmarcados por códigos de éticas y códigos deontológicos, la omisión puede ser un error grave para un Contador Público, ya que su opinión será referencia importante para la toma de decisiones de una determinada situación financiera de cualquier índole, la misión real de un contador público es auditar y emitir una opinión sobre el trabajo realizado y si esa opinión es alterada por aspectos negativos de formación de ética, entonces se reflejaría el verdadero problema y cómo afectaría en todos los aspectos a la sociedad.

Cabe destacar, que actuar éticamente implica hacerlo siempre acorde con las normas de comportamiento impuestas por la sociedad en un momento determinado. La actuación ética pasa por el conocimiento de las normas y principios existentes además del comportamiento del sujeto frente a estas normas. En este sentido, cuando se trata del ejercicio profesional de alguna carrera, como la de Contaduría Pública, se refiere a la ética profesional o deontología y se relaciona con el conjunto de normas y principios que rigen a un profesional en su actuación y frente situaciones laborales, permitiendo así la búsqueda de la excelencia profesional a través de la honestidad y responsabilidad.

En este orden de ideas, la deontología profesional hace referencia al conjunto de principios y reglas éticas que regulan y guían una actividad profesional. Estas normas determinan los deberes mínimamente exigibles a los profesionales en el desempeño de su actividad. Por este motivo, suele ser el propio colectivo profesional quién determina dichas normas $y$, a su vez, se encarga de recogerlas por escrito en los códigos deontológicos. A día de hoy, prácticamente todas las profesiones han desarrollado sus propios códigos y, en este sentido, puede hablarse de una deontología profesional periodística, de una deontología profesional médica, deontología profesional de los abogados, entre otras.

De la misma forma, la deontología analiza los deberes internos del individuo; es 
decir, aquello que debe hacer o evitar según lo que dicta su conciencia. Los valores compartidos y aceptados por la ética son recogidos por los códigos deontológicos. Es por eso, que el Contador Público, como tal, debe cimentar su reputación en la honestidad, laboriosidad y capacidad profesional, y no en el mercantilismo, ni expansionismo sin escrúpulos. Con el correr de los años han ocurrido una cantidad innumerable de fraudes financieros alrededor del mundo, todo esto a la falta de enfoque deontológico en el ejercicio de las ciencias administrativas y contables.

La Contaduría Pública se ha distinguido por ser una profesión que tiene como fin satisfacer necesidades de la sociedad, mediante la medición, evaluación, ordenamiento, análisis e interpretación de la información financiera de las empresas o los individuos y la preparación de informes sobre la correspondiente situación financiera, sobre los cuales se basan las decisiones de los empresarios, inversionistas, acreedores, demás terceros interesados y el Estado acerca del futuro de dichos entes económicos.

Asimismo, los profesionales de la Ciencia Contable en particular, toman decisiones e inducen a otras personas a tomarlas, por lo que la información que producen debe reunir ciertas características tales como veracidad, confiabilidad, oportunidad, entre otras características que requieren de un profesional que conduzca sus servicios bajo la guía de virtudes morales como la prudencia, la justicia, la fortaleza y la templanza.

Sin embargo, la Association of Certified Fraud Examiners, organización anti-fraude más grande del mundo estableció en su reporte del año 2012, que los países latinoamericanos con la tasa más grande de fraudes financieros son; México, Brasil, Colombia y Venezuela. Dicho informe indica que en Venezuela de alguna manera, son más bien permisivos porque las leyes no abordan este tipo de fraude de manera tan eficaz como debería ser.

Como consecuencia de la competencia entre las empresas por el posicionamiento en el mercado, se han presentado casos en los que se ajustan los estados financieros para beneficios personales de una o varias personas en conjunto de tal manera hasta el punto que la transacción es fraudulenta, aprovechándose de las normativas contables, para presentar una situación ficticia y de acuerdo a las exigencias de quienes manejan la empresa. Esta situación ligada a la falta de empleos hace que los contadores cedan en cuanto a trabajos de esta categoría, situación que afecta su ética profesional. Basándose en esto, lo habitual es que esta profesión promueva deontología profesional, que hace referencia al conjunto de principios y reglas éticas que regulan y guían una actividad profesional.

Es de resaltar, que en la en la carrera de Contaduría Pública de la Facultad de 
Ciencias Económicas y Sociales de la Universidad de Carabobo, Valencia, Venezuela, no existe una materia que trate el tema específicamente de la Deontología Profesional, sino que el mismo es estudiado de manera transversal en diversas materias, diluyéndose la importancia que el mismo tiene en el perfil del egresado y su ejercicio profesional.

Es de suma importancia, que los sujetos en su proceso de formación académica, sean sensibilizados en el tema de la deontología profesional, pues de ello depende que la profesión tenga prestigio ante la sociedad al ejercerla con valores y principios en cada una de sus actuaciones. Por ello, el objetivo de este estudio fue proponer una estrategia académica basada en la Deontología Profesional para fortalecer las competencias axiológicas en la formación del estudiante de Contaduría Pública de la Universidad de Carabobo.

\section{MATERIALES Y MÉTODOS}

El diseño del estudio fue de campo, documental, de carácter no experimental. Se trata de una investigación documental de tipo proyecto factible, al tratarse de una propuesta de asignatura sobre Deontología Profesional para ser incorporada en los estudios de la carrera de Contaduría Pública en la Facultad de Ciencias Económicas y Sociales de la Universidad de Carabobo, Valencia, Venezuela.

Se trata de una propuesta para tratar de resolver un problema práctico o una necesidad que sea factible de realizar, tomando en consideración la necesidad detectada, beneficios, recursos humanos, técnicos, financieros, estudio de mercado, y beneficiarios.

La población objeto de estudio estuvo conformada por los alumnos pertenecientes al décimo semestre de la carrera de Contaduría Pública, de la Escuela de Administración Comercial y Contaduría Pública de la Universidad de Carabobo, campus Bárbula, los cuales contabilizaron un total de 60 individuos, a quienes se les aplicó un cuestionario con respuestas dicotómicas.

\section{RESULTADOS Y DISCUSIÓN}

A continuación se presenta el resultado de la aplicación del cuestionario a los estudiantes del décimo semestre de la carrera de Contaduría Pública de la Facultad de Ciencias Económicas y Sociales de la Universidad de Carabobo:

Tabla. 1 Frecuencia de estudiantes que conocen la ley del ejercicio del Contador Público. Ítem $\mathbf{N}^{\circ} \mathbf{1}$ ¿Conoce la Ley del ejercicio del Contador Público y su reglamento?

\begin{tabular}{ccc}
\hline ALTERNATIVAS & FRECUENCIAS & PORCENTAJE \\
\hline SI & 58 & $96,67 \%$ \\
NO & 2 & $3,33 \%$ \\
TOTAL & $\mathbf{6 0}$ & $\mathbf{1 0 0 \%}$ \\
\hline
\end{tabular}

Fuente: Ortiz, Vargas, Velásquez (2019) 
Este resultado demuestra que la mayoría de los estudiantes tienen conocimiento sobre la legislación que rige el ejercicio de la Contaduría Pública, asimismo como también los componentes que conforman el gremio y las actividades del que desarrolla el profesional.

Tabla 2. Frecuencia de estudiantes que conocen el Código de Ética del Contador Público Venezolano. Ítem $\mathbf{N}^{0} 2$ ¿Conoce Ud. el Código de Ética del Contador Público Venezolano?

\begin{tabular}{ccc}
\hline ALTERNATIVAS & FRECUENCIAS & PORCENTAJE \\
\hline SI & 57 & $95,00 \%$ \\
NO & 03 & $5,00 \%$ \\
TOTAL & $\mathbf{6 0}$ & $\mathbf{1 0 0 \%}$ \\
\hline
\end{tabular}

Fuente: Ortiz, Vargas, Velásquez (2019)

Lo anterior indica que la mayoría de los estudiantes tienen conocimiento sobre el Código de Ética del Contador Público Venezolano, cuales son las normas éticas a seguir, cuales los valores que guían la profesión y cuáles son las consecuencias de no cumplir dicha legislación.

Tabla 3. Frecuencia de estudiantes que opinan que conocen o saben que es la Deontología Profesional. Ítem $\mathbf{N}^{0} 3$ ¿Conoce Ud. el termino Deontología Profesional?

\begin{tabular}{ccc}
\hline ALTERNATIVAS & FRECUENCIAS & PORCENTAJE \\
\hline SI & 9 & $15,00 \%$ \\
NO & 51 & $85,99 \%$ \\
TOTAL & $\mathbf{6 0}$ & $\mathbf{1 0 0 \%}$ \\
\hline
\end{tabular}

Fuente: Ortiz, Vargas, Velásquez (2019)

Este resultado refleja que la mayoría de los estudiantes del 10mo semestre de Contaduría Pública desconocen la definición de la deontología profesional, lo cual es preocupante ya que para el nivel académico que presentan deberían de dominar a cabalidad el marco conceptual enmarcado en el Código de Ética del Contador Público Venezolano. 
Tabla 4. Frecuencia conocimientos sobre que es la Deontología Profesional. Ítem $\mathbf{N}^{\circ} \mathbf{4}$ La Deontología es la ciencia que estudia el deber ser, tanto del profesional como el de la cotidianidad. ¿Cree Ud. que debería recibir más conocimientos sobre esta ciencia?

\begin{tabular}{ccc}
\hline ALTERNATIVAS & FRECUENCIAS & PORCENTAJE \\
\hline SI & 52 & $86,67 \%$ \\
NO & 8 & $13,33 \%$ \\
TOTAL & $\mathbf{6 0}$ & $\mathbf{1 0 0 \%}$ \\
\hline
\end{tabular}

Fuente: Ortiz, Vargas, Velásquez (2019)

Este resultado refleja la alta motivación que poseen los estudiantes del 10mo semestre de Contaduría Pública por conocer a fondo sobre la Deontología Profesional y así ampliar sus conocimientos en base a la legislación que rige al Contador Público venezolano.

Tabla 5. Frecuencia de estudiantes que la diferencia entre Ética Profesional y Deontología Profesional. Ítem $\mathbf{N}^{0} \mathbf{5}$ ¿Conoce Ud. la diferencia entre Ética Profesional y Deontología Profesional?

\begin{tabular}{ccc}
\hline ALTERNATIVAS & FRECUENCIAS & PORCENTAJE \\
\hline SI & 17 & $28,33 \%$ \\
NO & 43 & $71,67 \%$ \\
TOTAL & $\mathbf{6 0}$ & $\mathbf{1 0 0 \%}$
\end{tabular}

Fuente: Ortiz, Vargas, Velásquez (2019)

Este resultado demuestra que la mayoría de los estudiantes solo tienen conocimientos sobre la ética profesional, sin embargo carecen de los mismos para determinar la discrepancia que existe entre los términos antes mencionados.

Tabla 6. Frecuencia conocimiento de los códigos deontológicos que rigen el Código de Ética del Contador Público Venezolano. Ítem $\mathbf{N}^{\circ} \mathbf{6}$ ¿Conoce cuáles son los códigos deontológicos que rigen el Código de Ética del Contador Público Venezolano?

\begin{tabular}{ccc}
\hline ALTERNATIVAS & FRECUENCIAS & PORCENTAJE \\
\hline SI & 17 & $28,33 \%$ \\
NO & 43 & $71,67 \%$ \\
TOTAL & $\mathbf{6 0}$ & $\mathbf{1 0 0 \%}$ \\
\hline
\end{tabular}

Fuente: Ortiz, Vargas, Velásquez (2019) 
Este resultado afirma que la mayoría de los estudiantes carecen de los conocimientos sobre los códigos deontológicos, lo que conlleva a inferir que no se les ha dado la suficiente información sobre la deontología profesional y los códigos deontológicos los cuales se encuentran inmersos en el Código de Ética del Contador Público Venezolano.

Tabla 7. Frecuencia ausencia de conocimientos sobre la deontología. Ítem $\mathbf{N}^{\circ 7}$ ¿Considera usted que la ausencia de los conocimientos sobre la deontología profesional podría afectar su desempeño en el ejercicio de su carrera?

\begin{tabular}{ccc}
\hline ALTERNATIVAS & FRECUENCIAS & PORCENTAJE \\
\hline SI & 28 & $46,67 \%$ \\
NO & 32 & $53,33 \%$ \\
TOTAL & $\mathbf{6 0}$ & $\mathbf{1 0 0 \%}$ \\
\hline
\end{tabular}

Fuente: Ortiz, Vargas, Velásquez (2019)

Este resultado es alarmante, ya que evidencia la falta de interés de los estudiantes sobre los conocimientos deontológicos, lo cual puede contraer consecuencias a futuro, ya que poseen un pensamiento individualista por sobre el bien del gremio.

Tabla 8. Frecuencia conocimiento de consecuencias del no cumplimiento de los principios deontológicos. Ítem $\mathbf{N}^{\circ} \mathbf{8}$ ¿Conoce las consecuencias que conllevan el no cumplimiento de los principios deontológicos?

\begin{tabular}{ccc}
\hline ALTERNATIVAS & FRECUENCIAS & PORCENTAJE \\
\hline SI & 5 & $8,33 \%$ \\
NO & 55 & $91,67 \%$ \\
TOTAL & $\mathbf{6 0}$ & $\mathbf{1 0 0 \%}$ \\
\hline
\end{tabular}

Fuente: Ortiz, Vargas, Velásquez (2019)

Este resultado evidencia la falta de interés de los estudiantes sobre los conocimientos deontológicos, lo cual puede contraer lo podrían sanciones graves como lo es la suspensión del ejercicio profesional, y cuyo desconocimiento de la legislación no exime de su cumplimiento. 
Tabla 9. Frecuencia de estudiantes que consideran la ética, la moral y los valores son importantes en el ejercicio de la Contaduría Pública. Ítem $\mathbf{N} \times \mathbf{9}$ ¿Cree Ud. que la ética, la moral y los valores son importantes en el ejercicio de la Contaduría Pública?

\begin{tabular}{ccc}
\hline ALTERNATIVAS & FRECUENCIAS & PORCENTAJE \\
\hline SI & 17 & $28,33 \%$ \\
NO & 43 & $71,67 \%$ \\
TOTAL & $\mathbf{6 0}$ & $\mathbf{1 0 0 \%}$ \\
\hline
\end{tabular}

Fuente: Ortiz, Vargas, Velásquez (2019)

En este resultado se puede evidenciar el desinterés y el desconocimiento de los estudiantes sobre los códigos deontológicos, ya que desconocen la importancia de los mismos, así como también las consecuencias con respecto al no cumplimiento de los códigos deontológicos que rigen el ejercicio de la Contaduría Pública.

Tabla 10. Frecuencia de estudiantes que consideran que la Deontología Profesional es aplicable al ejercicio de la Contaduría Pública. Ítem $\mathbf{N}^{\mathbf{0}} \mathbf{1 0}$ ¿Cree Ud. que la Deontología Profesional es aplicable al ejercicio de la Contaduría Pública?

\begin{tabular}{ccc}
\hline ALTERNATIVAS & FRECUENCIAS & PORCENTAJE \\
\hline SI & 17 & $28,33 \%$ \\
NO & 43 & $71,67 \%$ \\
TOTAL & $\mathbf{6 0}$ & $\mathbf{1 0 0 \%}$ \\
\hline
\end{tabular}

Fuente: Ortiz, Vargas, Velásquez (2019)

Este resultado nos indica que la mayoría de los estudiantes podrían tener dificultades en un futuro, ya que la deontología profesional se encuentra intrínseca en cada uno de los profesionales que ejercen la carrera de Contaduría Pública, y siempre se está en busca del beneficio del gremio.

Tabla 11. Frecuencia de estudiantes que consideran la Deontología Profesional podría reducir los fraudes financieros que se observan a diario en el ejercicio de la Contaduría Pública. Ítem $\mathbf{N}^{\mathbf{0} 11}$ ¿Cree Ud. que la Deontología Profesional podría reducir los fraudes financieros que se observan a diario en el ejercicio de la Contaduría Pública?

\begin{tabular}{ccc}
\hline ALTERNATIVAS & FRECUENCIAS & PORCENTAJE \\
\hline SI & 17 & $28,33 \%$ \\
NO & 43 & $71,67 \%$ \\
TOTAL & $\mathbf{6 0}$ & $\mathbf{1 0 0 \%}$ \\
\hline
\end{tabular}

Fuente: Ortiz, Vargas, Velásquez (2019) 
Este resultado nos afianza que la falta de conocimientos sobre la deontología profesional, puede llevarlos a incurrir en fraudes financieros, ya que se evidencia que el pensamiento individual solapa al pensamiento colectivo.

Tabla 12. Frecuencia de estudiantes que consideran la Deontología Profesional es fundamental para triunfar tanto es la vida cotidiana como en el ejercicio profesional. Ítem No12 ¿Cree Ud. que la Deontología Profesional es fundamental para triunfar tanto es la vida cotidiana como en el ejercicio profesional?

\begin{tabular}{ccc}
\hline ALTERNATIVAS & FRECUENCIAS & PORCENTAJE \\
\hline SI & 41 & $68,33 \%$ \\
NO & 19 & $31,67 \%$ \\
TOTAL & $\mathbf{6 0}$ & $\mathbf{1 0 0 \%}$ \\
\hline
\end{tabular}

Fuente: Ortiz, Vargas, Velásquez (2019)

Con este resultado se da a entenderla importancia que le dan los estudiantes a los principios deontológicos para el crecimiento tanto personal como profesional.

Tabla 13. Frecuencia de estudiantes que consideran que los principios deontológicos forman la base de la confianza entre el cliente y quien presta el servicio. Ítem $\mathbf{N}^{\circ} \mathbf{1 3}$ ¿Cree Ud. que los principios deontológicos forman la base de la confianza entre el cliente y quien presta el servicio?

\begin{tabular}{ccc}
\hline ALTERNATIVAS & FRECUENCIAS & PORCENTAJE \\
\hline SI & 28 & $46,67 \%$ \\
NO & 32 & $53,33 \%$ \\
TOTAL & $\mathbf{6 0}$ & $\mathbf{1 0 0 \%}$ \\
\hline
\end{tabular}

Fuente: Ortiz, Vargas, Velásquez (2019)

La importancia de la utilidad de los códigos deontológicos se evidencia en este ítem, ya que la Contaduría Pública por definición es una profesión de libre ejercicio, lo que nos hace tratar directamente con el cliente al cual debemos mostrarle confianza y seguridad en el servicio que se le ofrecerá. 
Tabla 14. Frecuencia de estudiantes que consideran que se ha perdido la confianza en el Contador Público por la falta de valores éticos y deontológicos. Ítem $\mathbf{N}^{\mathbf{0}} \mathbf{1 4}$ La Contaduría Pública es una profesión la cual el cliente debe tener la total confianza de quien le suministra y le analiza la información de la entidad ¿Cree Ud. que se ha perdido la confianza en el Contador Público por la falta de valores éticos y deontológicos?

\begin{tabular}{ccc}
\hline ALTERNATIVAS & FRECUENCIAS & PORCENTAJE \\
\hline SI & 51 & $85 \%$ \\
NO & 9 & $15 \%$ \\
TOTAL & $\mathbf{6 0}$ & $\mathbf{1 0 0 \%}$ \\
\hline
\end{tabular}

Fuente: Ortiz, Vargas, Velásquez (2019)

Los grandes fraudes financieros actuales han dejado a la Contaduría Pública como una de las profesiones con mayor mala praxis, esto viene a raíz de la falta de conocimiento de los códigos éticos y deontológicos que nos rigen el ejercicio de la profesión, además de la falta de interés en la adquisición de dichos conocimientos, ya que se denotan como inservibles para el ejercicio profesional.

Tabla 15. Frecuencia de estudiantes que consideran han recibido por parte de la Universidad de Carabobo, los conocimientos pertinentes a la Deontología Profesional. Ítem $\mathbf{N}^{\circ} 15$ ¿Considera Ud. que ha recibido por parte de la Universidad de Carabobo, los conocimientos pertinentes a la Deontología Profesional?

\begin{tabular}{ccc}
\hline ALTERNATIVAS & FRECUENCIAS & PORCENTAJE \\
\hline SI & 5 & $8,33 \%$ \\
NO & 55 & $91,67 \%$ \\
TOTAL & $\mathbf{6 0}$ & $\mathbf{1 0 0 \%}$ \\
\hline
\end{tabular}

Fuente: Ortiz, Vargas, Velásquez (2019)

Se destaca en este los estudiantes entran al ejercicio profesional sin los conocimientos respectivos sobre los códigos deontológicos, lo que implica que en un futuro cercano podría incurrir con alguna falta a la legislación que rige el ejercicio profesional. 
Tabla 16. Frecuencia obligatoriedad asignatura deontología. Ítem $\mathbf{N}^{\circ} \mathbf{1 6}$ ¿Considera que la Deontología Profesional debería ser una asignatura obligatoria dentro del pensum de estudios de la carrera de Contaduría Pública de la Facultad de Ciencias Económicas y Sociales de la Universidad de Carabobo?

\begin{tabular}{ccc}
\hline ALTERNATIVAS & FRECUENCIAS & PORCENTAJE \\
\hline SI & 59 & $98,33 \%$ \\
NO & 1 & $1,67 \%$ \\
TOTAL & $\mathbf{6 0}$ & $\mathbf{1 0 0 \%}$ \\
\hline
\end{tabular}

Fuente: Ortiz, Vargas, Velásquez (2019)

Con este resultado se evidencia la necesidad de la apertura de una asignatura donde se impartan los conocimientos relacionados a la deontología profesional y a los códigos deontológicos.

\section{La propuesta}

Con base en los resultados anteriormente expresados, se propone la inclusión de la asignatura denominada "Deontología profesional en el ejercicio de la Contaduría Pública" en el pensum de estudios para la carrera de Contaduría Pública en la Facultad de Ciencias Económicas y Sociales de la Universidad de Carabobo. Una asignatura referente a la Deontología Profesional, con el fin de expandir los conocimientos, y así generar la formación del profesional integro en las ciencias contables que egresa de esta prestigiosa casa de estudios.

\section{Objetivo de la Propuesta}

A la luz de los resultados obtenidos, lo cual reflejó la carencia de conocimientos sobre Deontología Profesional en el ejercicio de la Contaduría Pública, cobra importancia dentro de la estructura curricular de la carrera de Contaduría Pública de la Universidad del Valle, debido a que permite que los estudiantes superen las visiones tradicionales de la Contaduría sólo como una técnica de registro, sino que existen variables cualitativas dentro de la misma que también son de suma importancia para su formación.

Las estructuras curriculares de los programas de contaduría pública han insistido en separar, en términos del diseño de dichas estructuras, las consideraciones teóricas y prácticas alrededor de la contabilidad. En este sentido, el Programa Académico de Contaduría Pública de la Universidad de Carabobo refleja de esta dinámica, orienta, por un lado, la práctica pedagógica a la aprehensión del ámbito instrumental de la contabilidad financiera (para lo cual construye una amplia gama de asignaturas que cumplen con este propósito) y, por el otro, no sitúa una asignatura cuya característica principal esa ampliar los conocimientos del estudiante con respecto a la Deontología Profesional en el ejercicio de la Contaduría Pública.

En atención a esto, se ha configurado como propuesta un programa de la materia Deontología profesional en el ejercicio de la Contaduría Pública, bajo los siguientes términos: 
Nombre de la asignatura:

Deontología profesional en el ejercicio de la Contaduría Pública

Escuela: Contaduría Pública Ciclo: Profesional Nivel:6

Horas semanales: 3

Síntesis de conocimientos previos:

Enfoque sistémico, principios de contabilidad generalmente aceptados

Tipo: Obligatoria

Departamento: Contabilidad

\section{OBJETIVOS Y COMPETENCIAS}

Objetivo General de la Asignatura: Al finalizar la asignatura el alumno estará en capacidad de comprender, valorar y manejar todos los conceptos básicos sobre la Deontología Profesional y su importancia en el ejercicio de la Contaduría Pública. Además podrá identificar los códigos y principios deontológicos que rigen el ejercicio profesional mediante el Código de Ética del Contador Público Venezolano. También podrá analizar e interpretar la importancia sobre la aplicación de los códigos deontológicos en el ejercicio de la Contaduría Pública.

\section{Objetivos de aprendizaje:}

1) Conocer el Código de Ética del Contador Público Venezolano (2012) aplicable al ejercicio de la contaduría pública así como los aspectos referidos a deontología profesional de las normativas internacionales.

2) Conocer los valores axiológicos para formar los criterios apropiados en la toma de decisiones en situaciones complejas y/o ambiguas que no estén articuladas de forma explícita dentro de los Códigos, por ser actividades muy concretas y específicas para un caso determinado o por ser actividades nuevas que aún no han sido desarrolladas suficientemente por el colegio profesional.

3) Sensibilizar al estudiante para que actúe de forma acorde a los criterios éticos y legales en los distintos ámbitos de actuación del Contador Público.

4) Fomentar la reflexión sobre los valores tanto sociales o culturales como personales e interpersonales y el desarrollo de actitudes morales consecuentes con los valores adoptados.

5) Fomentar el desarrollo en los estudiantes de actitudes de respeto e implicación o complacencia con las normas éticas que tienen que ver con la práctica contable tanto en su vertiente de intervención como de investigación.

6) Desarrollar en el estudiante el convencimiento de mantener un ejercicio profesional responsable y de calidad, el mantenimiento de unos altos estándares éticos es tan relevante como una alta formación teórico/ técnica.

7) Desarrollar una actitud de respeto hacia la pluralidad teórica y metodológica de la Contaduría Pública, siempre y cuando se encuentren dentro de los límites del conocimiento científico y legal vigente. 


\section{Nombre de la asignatura:}

Deontología profesional en el ejercicio de la Contaduría Pública

\section{SINOPSIS DEL CONTENIDO}

Tema 1. La deontología profesional en el Contador Público.

Tema 2. El perfil y las áreas de actuación del Contador Público.

Tema 3. Responsabilidades legales de los principios deontológicos. Responsabilidades penales, civiles y administrativas del Contador Público en el ejercicio de su actividad profesional.

Tema 4. Códigos deontológicos. Instrumentos legales y normas éticas que rigen el ejercicio profesional del Contador Público en el ámbito nacional e internacional.

Tema 5. Sociedades profesionales internacionales relacionadas con la Contaduría Pública

\section{CONCLUSIONES}

Es de suma relevancia la importancia dada por los encuestados al proceso de formación recibido o no en el área. Esto ha supuesto la importancia desde los alumnos por la inclusión en el grado de conocimientos específicos del área ética y deontología profesional, percibiendo así el vacío en la preparación de un saber-hacer ético que ayude a la toma de decisiones profesionales futuras.

En relación a la teoría presentada, y la información recolectada por las encuestas, se concluye que la mayoría de los estudiantes del décimo semestre de Contaduría Pública de la Universidad de Carabobo no manejan ni conocen que es la deontología profesional ni los códigos deontológicos ni su importancia dentro del ejercicio profesional de la Contaduría Pública, ya que su desconocimiento los pueden llevar a realizar actos nada propicios, los cuales pueden conducir a no lograr los objetivos ni trabajos encomendados de manera responsable, justa y honesta.

El desconocimiento de temáticas y problemáticas ético morales generales $\mathrm{y}$ particulares de la profesión es uno de las mayores debilidades del Contador Público Venezolano. La manifestación recurrente de esta cuestión ha evidenciado cierta desinformación y/o falta de interés respecto de búsqueda de fuentes especializadas al respecto. Sin embargo, una vez analizado los resultados del instrumento se pone de manifiesto un creciente interés por estos temas.

Para desarrollar la estrategia académica antes mostrada se propone lo siguiente:

Fortalecer, a partir de los inicios de la formación profesional en la Facultad de Ciencias Económicas y Sociales, la enseñanza sobre la deontología profesional, códigos deontológicos y valores éticos en todos los 
aspectos a los cuales se van a enfrentar los futuros profesionales de la carrera de Contaduría Pública, a fin que pueda obtener una educación superior integral bajo la premisa de una formación con los códigos deontológicos adecuados para el crecimiento tanto del individuo, como el gremio; en consecuencia generar una mejor armonía en la sociedad.

Enaltecer el aprendizaje de los códigos deontológicos en aquellos estudiantes que sienten indiferencia hacia los mismos, que demostraron que no perciben en su formación la enseñanza de los principios de la deontología profesional para así constituir una herramienta para desempeñarse a cabalidad de manera congruente con las exigencias actuales que presenta el ejercicio profesional de la Contaduría Pública en Venezuela.

Instaurar convenios con el Colegio de Contadores Públicos de Carabobo, La Federación de Colegios de Contadores Públicos y la Universidad de Carabobo con la finalidad de preparar cursos, talleres, charlas, debates que permitan la formación integral del Contador Público, con el fin del enriquecer la sana competitividad que acarrea actualmente el ejercicio de la Contaduría Pública.
Concientizar a los estudiantes en su formación como profesional de la Contaduría Pública sobre el significado y la importancia que tienen los códigos deontológicos en el desarrollo del ejercicio profesional de la carrera.

\section{REFERENCIAS}

Association of Certified Fraud Examiners (2012. Informe a las Naciones 2012. Disponible en: https://www.acfe.com/rttn-2012.aspx.

Consulta realizada 12/01/2020

Escobar G. (2000). Ética. (Cuarta Edición). Editorial Mc Graw Hill. Ciudad de México, México

Federación de Colegios de Contadores

Públicos de Venezuela. (2013). Publicaciones Legales y Reglamentarias PLR-3: Código de Ética para regular el ejercicio Profesional del Contador Público. Fondo Editorial de Contadores Público Venezolano. Caracas

Guillermo González-Vallés Saco (2014).

Deontología. Página web: http://deontologia.org/pagina1.html.

Consulta realizada 15/01/2020

\section{Ley de Ejercicio de la Contaduría Pública}

(PLR-1). (1973, Diciembre 5). Gaceta Oficial de la República de Venezuela, 30.273, Diciembre 5, 1973. Caracas 